\title{
Unification of gauge and gravity Chern-Simons theories in 3-D space-time
}

\author{
Chireen A. Saghir ${ }^{\mathrm{a}}$, Laurence W. Shamseddine \\ Physics Department, American University of Beirut, Beirut, Lebanon
}

Received: 13 September 2017 / Accepted: 4 November 2017 / Published online: 20 November 2017

(C) The Author(s) 2017. This article is an open access publication

\begin{abstract}
Chamseddine and Mukhanov showed that gravity and gauge theories could be unified in one geometric construction provided that a metricity condition is imposed on the vielbein. In this paper we are going to show that by enlarging the gauge group we are able to unify Chern-Simons gauge theory and Chern-Simons gravity in 3-D space-time. Such a unification leads to the quantization of the coefficients for both Chern-Simons terms for compact groups but not for non-compact groups. Moreover, it leads to a topological invariant quantity of the 3-dimensional space-time manifold on which they are defined.
\end{abstract}

\section{Introduction}

Topological Field Theories (TFTs) are metric independent theories that define topological invariants of the manifold M. In general, we can distinguish between a topological field theory of Schwarz type and of Witten or cohomological type. In Schwarz type theories the action is explicitly independent on the metric

$\frac{\delta S}{\delta g_{\mu \nu}}=0$

Examples of such theories are Chern-Simons theories and BF theories. The other type is Witten type topological field theories like the Donaldson-Witten theory, which was formulated by Witten in 1988 [1] and is used to compute the Donaldson invariant.

In a ground-breaking work [2], Witten (1989) showed that Chern-Simon gauge theories provide a physical description of a wide class of invariants of 3-manifolds and of knots and links in this manifold. The Chern-Simon action with a

a e-mail: cas25@mail.aub.edu compact and simple gauge group $\mathrm{G}=\mathrm{SU}(\mathrm{N})$ on a generic 3-manifold is defined by

$S=\frac{k}{4 \pi} \int \operatorname{Tr}\left(A \wedge d A+\frac{2}{3} A \wedge A \wedge A\right)$.

Here $\mathrm{k}$ is the coupling constant and A is a G-gauge connection on the trivial bundle on $\mathrm{M}$.

The partition function

$Z(M)=\int D A e^{i S}$

is really a topological invariant of the manifold $\mathrm{M}$ as was proved by Witten [2]. Moreover, if L is a collection of oriented and non-intersecting knots $C_{i}$, we have the partition function

$Z(M, L)=\int D A \exp (i S) \prod_{i=1}^{r} W_{R_{i}}\left(C_{i}\right)$

where

$W_{R}(C)=\operatorname{Tr}_{R} P \exp \int_{C} A$

represents a certain type of knot invariant called the Jones polynomial.

In general, Chern-Simons theories in the 3-D case exist for both gauge theory and gravity. Several works have been published to investigate the importance of ChernSimons theories. In [3], Deser, Jackiw and Templeton had shown that adding a topological Chern-Simons term to both 3-dimensional Yang-Mills and gravity theories generate masses of the corresponding gauge particles and alters its content. Moreover, in [4] Chamseddine and Frohlich showed that the Lorentz and mixed Lorentz-Weyl anomaly, but not the pure Weyl anomaly, of the 2-dimensional chiral bosons and fermions can be cancelled by the anomalies of the 3dimensional gravitational Chern-Simons action. Quantum 
gravity in $2+1$ dimensions has also been studied [5]. In a recent work [6], Chamseddine and Mukhanov had shown that by enlarging the tangent group to $\mathrm{SO}(1,13)$ we are able to unify gravity and gauge interactions using a procedure that is applicable for an arbitrary dimension of the tangent space.

In this letter we show how to unify gauge and gravity Chern-Simons theories in 3-D space-time. Such a unification has several interesting results. First, we will be able to investigate the quantization of the coupling constants for both theories (gauge and gravity) with Lorentzian and Euclidean signature. Second, we will obtain the resulting topological invariant quantity of the 3-D space-time manifold.

\section{Unification of both Chern-Simons theories}

Before presenting our work, we would like to review the work of Chamseddine and Mukhanov [6]. In this work, it has been shown that it is possible to unify Yang-Mills gauge theories with gravity in terms of higher-dimensional gauged Lorentz groups of the tangent space. The 4-dimensional manifold is spanned by the coordinate basis $e_{\alpha}$ where $\alpha=1, \ldots, 4$, and the $N \geqslant 4$-dimensional tangent space is spanned by $v_{A}$, where $A=1,2, \ldots, N$. The two basis vectors are related by the vielbeins $e_{\alpha}^{A}$ through $e_{\alpha}=e_{\alpha}^{A} v_{A} . \mathbf{n}_{\hat{J}}$ constitute a set of $N-4$ orthonormal vectors, orthogonal to the subspace spanned by $\mathbf{e}_{\alpha}$, that is, $\mathbf{n}_{\hat{J}} \cdot \mathbf{e}_{\alpha}=0$ and $\mathbf{n}_{\hat{J}} \cdot \mathbf{n}_{\hat{I}}=\delta_{\hat{J} \hat{I}}$, where $\hat{J}, \hat{I}=5,6, \ldots, N$. The vectors $\mathbf{n}_{\hat{J}}, \mathbf{e}_{\alpha}$ form a complete basis in tangent space and therefore $\mathbf{v}_{A}$ can be expanded as

$\mathbf{v}_{A}=v_{A}^{\alpha} \mathbf{e}_{\alpha}+n_{A}^{\hat{J}} \mathbf{n}_{\hat{J}}$

The affine connection $\Gamma_{\alpha \beta}^{v}$ and the spin connection $w_{\beta A}^{B}$ defines the parallel transport for coordinate basis and vielbeins, respectively,

$\nabla_{\mathbf{e}_{\beta}} \mathbf{e}_{\alpha} \equiv \nabla_{\beta} \mathbf{e}_{\alpha}=\Gamma_{\alpha \beta}^{v} \mathbf{e}_{v}, \quad \nabla_{\beta} \mathbf{v}_{A}=-\omega_{\beta A}{ }^{B} \mathbf{v}_{B}$

The two connections are related by the metricity condition,

$\partial_{\beta} e_{A \alpha}=-\omega_{\beta A}{ }^{B} e_{B \alpha}+\Gamma_{\alpha \beta}^{\nu} e_{A \nu}$

$R_{\alpha \beta}^{A B}(w)$ is the Riemann curvature tensor of the spin connection of the group $\mathrm{SO}(1, \mathrm{~N}-1)$ defined by

$R_{\alpha \beta}^{A B}(w)=\partial_{\alpha} w_{\beta}^{A B}-\partial_{\beta} w_{\alpha}^{A B}+w_{\alpha}^{A C} w_{\beta C}^{B}-w_{\beta}^{A C} w_{\alpha C}^{B}$

and $R_{\gamma \alpha \beta}^{\rho}(\Gamma)$ is that of affine connection of the group $\operatorname{SO}(1,3)$ defined by

$R_{\gamma \alpha \beta}^{\rho}(\Gamma)=\partial_{\alpha} \Gamma_{\beta \gamma}^{\rho}-\partial_{\beta} \Gamma_{\alpha \gamma}^{\rho}+\Gamma_{\alpha \kappa}^{\rho} \Gamma_{\beta \gamma}^{\kappa}-\Gamma_{\beta \kappa}^{\rho} \Gamma_{\alpha \gamma}^{\kappa}$.
The spin connection curvature $R_{\alpha \beta}{ }^{A B}(\omega)$ can be expressed in terms of the affine connection $R_{\gamma \alpha \beta}^{\sigma}(\Gamma)$ by

$R_{\alpha \beta}{ }^{A B}(\omega)=R_{\alpha \beta}^{A C}(\omega) n_{C}^{\hat{I}} n_{\hat{I}}^{B}+R_{\gamma \alpha \beta}^{\rho}(\Gamma) e_{\rho}^{A} e^{B \gamma}$.

Next, it is shown that the first term on the right hand side of this equation can be entirely expressed in terms of the spin connections defining the parallel transport of vectors $\mathbf{n}_{\hat{J}}$ in the subspace of tangent space orthogonal to those part spanned by the four coordinate basis vectors $\mathbf{e}_{\alpha}$. These connections are denoted by $A_{\beta \hat{J}} \hat{I}$. Finally, Eq. (11) can be written as

$R_{\alpha \beta}^{A B}(w)=F_{\alpha \beta}^{\hat{J} \hat{I}} n_{\hat{J}}^{A} n_{\hat{I}}^{B}+R_{\gamma \alpha \beta}^{\rho}(\Gamma) e_{\rho}^{A} e^{B \gamma}$

$F_{\alpha \beta}^{\hat{J} \hat{I}}$ is the curvature tensor or field strength of the remaining $\mathrm{SO}(\mathrm{N}-4)$ group,

$F_{\alpha \beta}^{\hat{I} \hat{J}}(A)=\partial_{\alpha} A_{\beta}^{\hat{I} \hat{J}}-\partial_{\beta} A_{\alpha}^{\hat{I} \hat{J}}+A_{\alpha}^{\hat{I} \hat{L}} A_{\beta \hat{L}}^{\hat{J}}-A_{\beta}^{\hat{I} \hat{L}} A_{\alpha \hat{L}}^{\hat{J}}$.

The first term to the right hand side of Eq. (12) correspond to $\mathrm{SO}(\mathrm{N}-4)$ gauge theory and the second one to $\mathrm{SO}(1,3)$ gravity theory.

The easiest way to understand the above results which showed that the $S O(1, N-1)$ invariants split into $S O(1,3)$ and $S O(N-4)$ invariants, is to work in a special gauge [6]. We first split the constraint Eq. (8) for $A=a=1, \ldots, 4$ and $A=\hat{I}=5, \ldots, N$ :

$0=\partial_{\mu} e_{a}^{v}+\omega_{\mu a}^{b} e_{b}^{v}+\omega_{\mu a} \hat{I}_{\hat{I}}^{v}+\Gamma_{\mu \rho}^{\nu} e_{a}^{\rho}$,

$0=\partial_{\mu} e_{\hat{I}}^{\nu}+\omega_{\mu \hat{I}}^{a} e_{a}^{v}+\omega_{\mu \hat{I}}^{\hat{J}} e_{\hat{J}}^{v}+\Gamma_{\mu \rho}^{v} e_{\hat{J}}^{\rho}$.

The vielbeins $e_{A}^{\mu}$ transform under $S O(1, N-1)$ transformations according to

$e_{A}^{\mu} \rightarrow \tilde{e}_{A}^{\mu}=\Lambda_{A B} e^{\mu B}$

In particular,

$e_{\hat{I}}^{\mu} \rightarrow \tilde{e}_{\hat{I}}^{\mu}=\Lambda_{\hat{I} a} e^{\mu a}+\Lambda_{\hat{I} \hat{J}} e^{\mu \hat{J}}$

The action, by construction, is invariant under $S O(1, N-1)$ rotations. Thus, it is possible to use the gauge invariance and the freedom in the choice of gauge parameters $\Lambda_{\hat{I} a}$ to set $e_{\hat{I}}^{\mu}$ to zero

$e_{\hat{I}}^{\mu}=0$

This leaves the gauge parameters $\Lambda_{a b}$ and $\Lambda_{\hat{I} \hat{J}}$ arbitrary, corresponding to invariance under the subgroup $S O(1,3) \times$ 
$S O(N-4)$. With this gauge choice we see that Eq. (15) implies

$\omega_{\mu \hat{I}}^{a}=0$

assuming that $e_{a}^{\mu}$ is invertible. The remaining equation (14) can now be solved to give the usual expression for $\omega_{\mu a}{ }^{b}$ in terms of $e_{a}^{\mu}$ and its derivative. In this special gauge $\omega_{\mu \hat{I}} \hat{J}=$ $A_{\mu \hat{I}}^{\hat{J}}$ and

$R_{\mu \nu}^{a \hat{I}}=0$

while nonvanishing components of the curvature $R_{\mu \nu}{ }^{a b}$ and $R_{\mu \nu} \hat{I} \hat{J}$ are responsible for the gravity and gauge fields, respectively.

The realistic group which can allow us to unify all known interactions in one family is $\mathrm{SO}(1,13)$ and leads to Einstein gravity with the $\mathrm{SO}(10)$ gauge group being, however, not entirely equivalent to the $\mathrm{SO}(10)$ grand unified theory.

Returning to our work, their are two ways to proceed : We can start by proving that the Pontryagin density in 4-D spacetime of the larger group $\mathrm{SO}(\mathrm{N})$ splits into one corresponding to gauge theory and another for gravity. Then we relate each Pontryagin density to its corresponding Chern-Simons term in 3-D space-time. The second way is to start directly in 3D space-time and prove that the Chern-Simons term for the larger groups splits into two terms one for gauge theory and the other for gravity.

\subsection{Pontryagin densities}

The starting points for the construction of the Chern-Simons terms are objects called Chern-Pontryagin densities. On a 2n-dimensional manifold, these are of the form

$P^{2 n} \propto \epsilon^{\mu_{1} \mu_{2} \ldots \mu_{2 n}} \operatorname{Tr}\left(F_{\mu_{1} \mu_{2}} \ldots F_{\mu_{2 n-1} \mu_{2 n}}\right)$

where $\mathrm{F}$ (field strength) is the curvature 2-form $(d A+A \wedge A)$ of some $\mathrm{G}$-connection ( $\mathrm{G}$ is the gauge group). These are gauge-invariant, closed, and their integral over the manifold $\mathrm{M}$ (compact, no boundary) is an integer which is a topological invariant. These sorts of invariants are examples of characteristic classes.

The Pontryagin density for gauge theory is

$P_{4}=-\frac{1}{16 \pi^{2}} \operatorname{Tr}\left({ }^{*} F^{\mu \nu} F_{\mu \nu}\right)$

where

$F_{\mu \nu}^{a}=\partial_{\mu} A_{\nu}^{a}-\partial_{\nu} A_{\mu}^{a}+f^{a b c} A_{\mu b} A_{\nu c}$ and that for gravity is the (Hirzebruch-Pontryagin) given by

$* R R=\frac{1}{2} \epsilon^{\mu \nu \alpha \beta} R_{\mu \nu \rho \sigma} R_{\alpha \beta}^{\rho \sigma}$.

Using Eq. (12), we can write the Pontryagin density for the large group $\mathrm{SO}(\mathrm{N})$ in 4-D

$$
\begin{aligned}
& \frac{1}{2} \epsilon^{\mu \nu \alpha \beta} R_{\mu \nu}^{A B} R_{\alpha \beta A B} \\
& =\frac{1}{2} \epsilon^{\mu \nu \alpha \beta}\left(F_{\mu \nu}^{\hat{J} \hat{I}} n_{\hat{J}}^{A} n_{\hat{I}}^{B}+R_{\gamma \mu \nu}^{\rho} e_{\rho}^{A} e^{B \gamma}\right)\left(F_{\alpha \beta}^{\hat{K} \hat{L}} n_{\hat{J} A} n_{\hat{L} B}\right. \\
& \left.\quad+R_{\sigma \alpha \beta}^{\delta} e_{\delta A} e_{B}^{\sigma}\right) .
\end{aligned}
$$

Using the relation $n_{\hat{J}}^{A} e_{A}^{\alpha}=0$ [6], we deduce that the mixed terms vanish and we are left with the Pontryagin densities corresponding to gauge theory and gravity,

$$
\begin{aligned}
\frac{1}{2} \epsilon^{\mu \nu \alpha \beta} R_{\mu \nu}^{A B}, R_{\alpha \beta A B}= & \frac{1}{2} \epsilon^{\mu \nu \alpha \beta} F_{\mu \nu \hat{K} \hat{L}} F_{\alpha \beta}^{\hat{K} \hat{L}} \\
& +\frac{1}{2} \epsilon^{\mu \nu \alpha \beta} R_{\mu \nu}^{A B} R_{\alpha \beta A B}
\end{aligned}
$$

We can deduce that in 4-D space-time, the Pontryagin densities for both gauge theory and gravity can be unified.

We are interested in showing the unification in 3-D spacetime. The Chern-Pontryagin densities in 4-D are the exterior derivatives of the Chern-Simons entities in 3-D.

$$
\begin{aligned}
P_{4}=\operatorname{Tr}(F \wedge F) & =\operatorname{Tr}((d A+A \wedge A)(d A+A \wedge A)) \\
& =\operatorname{Tr}\left(d\left(A d A+A^{3}\right)\right)=\operatorname{Tr}\left(d w_{3}\right),
\end{aligned}
$$

where $\operatorname{Tr}\left(A^{4}\right)=0$.

We have $I_{C S}=\int w_{3}$ in $3-\mathrm{D}$. Then

$\int_{M_{4}} P_{4}=\int_{M_{4}} d w_{3}=\int_{\partial M_{4}} w_{3}$.

Based on this, we can easily translate the 4-D unification $\int_{M_{4}} P_{4}=\int_{M_{4}} P_{4}^{\text {gauge }}+\int_{M_{4}} P_{4}^{\text {gravity }}$ into a 3 -D one of the corresponding Chern-Simons terms

$\int_{M_{3}} w_{3}=\int_{M_{3}} w_{3}^{\text {gauge }}+\int_{M_{3}} w_{3}^{\text {gravity }}$

where

$$
\begin{aligned}
& w_{3}^{\text {gauge }}=\epsilon^{i j k}\left(A_{i}^{a} \partial_{j} A_{k}^{a}+\frac{1}{3} f^{a b c} A_{i}^{a} A_{j}^{b} A_{k}^{c}\right), \\
& w_{3}^{\text {gravity }}=\epsilon^{i j k}\left(R_{i j a b} w_{k}^{a b}+\frac{2}{3} w_{i b}^{c} w_{j c}^{a} w_{k a}^{b}\right) .
\end{aligned}
$$




\subsection{3-D Chern-Simons terms}

Alternatively, we can show the unification by starting directly in 3-D space-time. The topological Chern-Simons term corresponding to the larger group $\mathrm{SO}(\mathrm{N})$ is

$I_{C S}=\frac{k}{4 \pi} \int \operatorname{Tr}\left(A \wedge d A+\frac{2}{3} A \wedge A \wedge A\right)$,

where $\mathrm{A}$ is the 1 -form connection of the group, $A=$ $d x^{\mu} \frac{1}{4} A_{\mu}^{A B} \Gamma_{A B}$ and $\mathrm{A}, \mathrm{B}, \mathrm{C}$ range from 1 to $\mathrm{N}$.

$\Gamma$-matrices is a set of $2^{D}$ matrices resulting from the repeated multiplication of the $\gamma$-matrices of the D-dimensional clifford algebra given by [7].

$\gamma_{a} \gamma_{b}+\gamma_{b} \gamma_{a}=2 \delta_{a b} I$

where $a, b$ ranges from 1 to $\mathrm{D}$.

Following the properties of $\Gamma$ matrices in an arbitrary space-time dimension [7], the Chern-Simons term becomes

$I_{C S}=\frac{k}{4 \pi} \int\left(A^{A C} d A^{C A}+\frac{2}{3} A^{A B} A^{B C} A^{C A}\right)$.

Now, we can split the indices A, B, C into a, b, c, which go from 1 to 3 , and $\hat{I}, \hat{J}, \hat{K}$, which range from 4 to $\mathrm{N}$. We get

$$
\begin{aligned}
I_{C S}= & \frac{k}{4 \pi} \int A^{A C} d A^{C A}+\frac{2}{3} A^{A B} A^{B C} A^{C A} \\
= & \frac{k}{4 \pi} \int A^{a b} d A^{b a}+\frac{2}{3} A^{a b} A^{b c} A^{c a} \\
& +\frac{k}{4 \pi} \int A^{\hat{I} \hat{J}} d A^{\hat{J} \hat{I}}+\frac{2}{3} A^{\hat{I} \hat{J}} A^{\hat{J} \hat{K}} A^{\hat{K} \hat{I}} .
\end{aligned}
$$

We notice that the first term in Eq. (34) corresponds to gravity Chern-Simons action and the second one to gauge ChernSimons action.

\section{Consequences of this unification}

\subsection{Quantization of the coefficient}

The coefficient $\mathrm{k}$ of the Chern-Simons term for compact simple groups is quantized [2]. To unify non-abelian ChernSimons gauge theory with the gravity Chern-Simons theory, we have two possibilities. For space-time with Euclidean signature, we can choose our group to be the compact $\mathrm{SO}(6)$ group, which will split into $\mathrm{SO}(3)$ for the gravity term and $\mathrm{SO}$ (3) for the gauge theory term. Such a choice leads to the quantization of the coupling constant $\mathrm{k}$ for the three terms, which must be the same. Thus, we get the quantization of the coupling constant of the gravity Chern-Simons term with the compact group $\mathrm{SO}(3)$. The other possibility is for manifolds with Lorentzian signature. We choose the non-compact group $\mathrm{SO}(1,5)$, which splits into $\mathrm{SO}(1,2)$ for gravity and $\mathrm{SO}(3)$ for gauge theory. The homotopy group of $\mathrm{SO}(1,5)$ is equal to that of $\mathrm{SO}(5)$,

$\pi_{5}(S O(1,5))=\pi_{5}(S O(5))=Z_{2}$,

the coefficient $\mathrm{k}$ must not be quantized as the winding number is not sensitive to torsion and vanishes [8].

\subsection{Topological invariants}

A topological field theory is a theory that computes topological invariants. Witten [2] proved that, for the weak coupling limit (large $\mathrm{k}$ ), the partition function

$Z=\int D A \exp \left(\frac{i k}{4 \pi} \int_{M} \operatorname{Tr}\left(A d A+\frac{2}{3} A \wedge A \wedge A\right)\right.$

is a topological invariant quantity of the 3-D space-time manifold. We are going to proceed as Witten did. For the $\operatorname{SO}(6)$ group, the partition function $\mathrm{Z}$ splits into two parts,

$$
\begin{aligned}
Z & =\int D A \exp \left(i I_{C S}\right)=\int D A \exp \left(i I_{\text {gauge }}+i I_{\text {gravity }}\right) \\
& =\int D w \exp \left(i I_{\text {gravity }}\right) \int D B \exp \left(i I_{\text {gauge }}\right)=Z_{1} \cdot Z_{2}
\end{aligned}
$$

where the first term is the $\mathrm{SO}(1,2)$ gravity Chern-Simon term with $w$ as a gauge connection and the second term is the $\mathrm{SO}(3)$ gauge Chern-Simon term with B as a gauge field.

We consider the weak coupling limit of the gauge part. The weak coupling limit of $Z_{2}$ is given by

$Z_{2}=\sum_{\alpha} \mu\left(B^{\alpha}\right)$

where $\mu\left(B^{\alpha}\right)$ is a function of the flat connections, for which the curvature vanishes. We expand the gauge field $B_{i}=$ $B_{i}^{(\alpha)}+C_{i}$, and the Chern-Simon gauge action term becomes

$I_{C S}^{\text {gauge }}=k I\left(B^{\alpha}\right)+\frac{k}{4 \pi} \int_{M} \operatorname{Tr}(C \wedge D C)$

where $\mathrm{D}$ is the covariant derivative with respect to $B^{\alpha}$.

To carry out the Gaussian integral in Eq. (39), a gauge fixing is needed, which cannot be done without picking a metric. We choose such a metric to satisfy $D_{i} C^{i}=0$. The resulting ghost action becomes

$S_{G F}=\int_{M} \operatorname{Tr}\left(\phi D_{i} C^{i}+\bar{c} D_{i} D^{i} c\right)$

where $\phi$ is a Lagrangian multiplier that enforces the gauge condition $D_{i} C^{i}=0$, and $\mathrm{c}, \bar{c}$ are anticommuting ghosts. 
Integrating out $\mathrm{C}, \phi, \mathrm{c}$, and $\bar{c}$, we get

$\exp \left(\frac{i \pi \eta\left(B^{\alpha}\right)}{2}\right) T_{\alpha}$

where $\eta\left(B^{\alpha}\right)$ is the "eta invariant" defined by

$\eta\left(B^{\alpha}\right)=\frac{1}{2} \lim _{s \rightarrow} \sum_{i} \operatorname{sign} \lambda_{i}\left|\lambda_{i}\right|^{-s}$

where the $\lambda_{i}$ are eigenvalues of operator $L_{i}$, the restriction of $* D_{B}+D_{B} *$ on odd forms, $T_{\alpha}$ is the torsion invariant of the flat connection $B^{(\alpha)}$. Using the Atiyah-Patodi-Singer index theorem, the partition function can be written as

$Z_{2}=\exp \left(i \frac{\pi}{2} \eta(0)\right) \sum_{\alpha} e^{i\left(k+c_{2}(G) / 2\right) I\left(B^{(\alpha)}\right)} \cdot T^{\alpha}$.

$\eta(0)$ is the eta invariant of the trivial gauge field and $c_{2}(G)$ is the Casimir operator of G. $\eta(0)$ is the only term that depends on the metric. Witten suggested that by adding a counter term, the partition function will be regularized and turned into a topological invariant quantity. This counter term must be a multiple of the gravitational Chern-Simons term. This term is already present in our case. Substituting the weak coupling limit of $Z_{2}$, Eq. (43), in Eq. ( 37) we get

$$
\begin{aligned}
Z & =\int D w \exp \left(i I_{\text {grav }}\right) \cdot Z_{2} \\
& =\int D w \exp \left(i I_{\text {grav }}\right) \exp \left(i \frac{\pi}{2} \eta(0)\right) \sum_{\alpha} e^{i\left(k+c_{2}(G) / 2\right) I\left(B^{(\alpha)}\right)} \cdot T^{\alpha}, \\
& =\int D w \exp \left(i\left(I_{\text {grav }}+\frac{\pi}{2} \eta(0)\right)\right) \sum_{\alpha} e^{i\left(k+c_{2}(G) / 2\right) I\left(B^{(\alpha)}\right)} T^{\alpha} .
\end{aligned}
$$

The gravitational Chern-Simons term added by Witten is already present in our case. So our partition function is a topological invariant quantity without the adding any term.

Summarizing, we have shown how gravity and gauge Chern-Simons theories could be unified in 3-D space-time. The coupling constant is quantized for compact groups but not for non-compact ones. Moreover, the partition function $\mathrm{Z}$ directly becomes a topological invariant quantity without the need of adding any term as Witten did [2]. It should be noted that such Chern-Simon theories admit local supersymmetric extensions. This is achieved by considering the gauging of supergroups, or by extending the space-time manifold to a supermanifold, or both [8]. We shall consider the graded groups that are extensions of our $\mathrm{SO}(1,5)$ group. Referring to [8], we could choose the supergroup

$O(6,1) \oplus S U(2),(8,2)$

where we can prove that the unification still exists for supersymmetric Chern-Simon theories. Details of this will be dealt with in future work.

Acknowledgements We would like to thank Professor Ali Chamseddine for suggesting the problem and for his many helpful discussions on the subject. We would like to thank the American University of Beirut (Faculty of Science) for support.

Open Access This article is distributed under the terms of the Creative Commons Attribution 4.0 International License (http://creativecomm ons.org/licenses/by/4.0/), which permits unrestricted use, distribution, and reproduction in any medium, provided you give appropriate credit to the original author(s) and the source, provide a link to the Creative Commons license, and indicate if changes were made.

Funded by SCOAP S $^{3}$.

\section{References}

1. S.K. Donaldson, J. Diff. Geom. 18, 279 (1983)

2. E. Witten, Commun. Math. Phys. 121, 351 (1989)

3. S. Deser, R. Jackiw, S. Templeton, Topologically massive gauge theories. Ann. Phys. 281, 409-449 (2000)

4. A.H. Chamseddine, J. Frohlich, Two-dimensional Lorentz-Weyl anomaly and gravitational Chern-Simons theory., Commun. Math. Phys. 147, 549 (1992)

5. Carlip, S.: Quantum Gravity in 2+1 Dimensions. Cambridge University Press. ISBN 0521545889

6. Chamseddine, A.H., Mukhanov, V.: On the unifcation of gravity and gauge interaction. J. High Energ. Phys. 2016, 20 (2016)

7. De Wit, B., Smith, J.: Field theory in particle physics. NorthHolland, Amsterdam. ISBN 0444869964

8. A.H. Chamseddine, Toplogical gravity and supergravity in various dimensions. Nuclear Phys. B 346, 213-234 (1990) 